\title{
Next-Generation Consumer Electronics for 6G Wireless Era
}

\author{
SYED JUNAID NAWAZ1', Senior Member, IEEE, \\ SHREE KRISHNA SHARMA ${ }^{2}$, Senior Member, IEEE, \\ MOHMAMMAD N. PATWARY ${ }^{3}$, Senior Member, IEEE, and \\ MD ASADUZZAMAN ${ }^{4}$, Senior Member, IEEE \\ ${ }^{1}$ Department of Electrical and Computer Engineering, COMSATS University Islamabad (CUI), Islamabad 45550, Pakistan. e-mail: junaidnawaz@ieee.org \\ ${ }^{2}$ SnT - securityandtrust.lu, University of Luxembourg, Kirchberg, Luxembourg 1855, Luxembourg. e-mail: shree.sharma@uni.lu \\ ${ }^{3}$ Faculty of Science \& Engineering, University of Wolverhampton, Wolverhampton WV1 1LY, UK. e-mail: patwary@wlv.ac.uk \\ ${ }^{4}$ School of Digital, Technologies and Arts, Staffordshire University, Stoke on Trent ST4 2DE, UK. e-mail: asad@ieee.org \\ Corresponding author: Syed Junaid Nawaz (e-mail: junaidnawaz@ieee.org).
}

\begin{abstract}
The upcoming beyond 5G (B5G)/6G wireless networks target various innovative technologies, services, and interfaces such as edge computing, ultra-reliable and low-latency communication (URLLC), backscatter communications, and TeraHertz (THz) technology-enabled inter-chip high-capacity communications, to name a few. Although there are ongoing advances in the system/network level, it is crucial to advance the device-level design to efficiently support these novel technologies by addressing various practical constraints in terms of power, computational capacity, and storage capacity limitations. This need for device-level innovation ultimately demands significant enhancements in today's consumer electronics (CE). Considering the contemporary latency requirements of CE applications (e.g., entertainment, gaming, etc), to enhance the commercial potential of "edge processing as service", it is envisioned that URLLC will further evolve as enhanced-URLLC (e-URLLC) in the B5G era. In this regard, this paper proposes a novel edge computing-enabled e-URLLC framework, named edge computing for CE (ECCE), to support advancements and to initiate discussions on the need for next-generation CE. Starting with the discussion on recent trends and advances in CE, the proposed framework and its importance in the $6 \mathrm{G}$ wireless era are described. Subsequently, several potential technologies and tools to enable the implementation of the proposed ECCE framework are identified along with some interesting open research topics and future recommendations.
\end{abstract}

INDEX TERMS 5G, 6G, B5G, consumer electronics, edge computing, URLLC,

\section{INTRODUCTION}

Towards enhancing the quality of human life experience, research in different areas is progressively contributing. Advancement in integrated circuits, information processing, and wireless communications towards boosting the number of transistors in a wafer area, speed of information processing, and rate of information exchange, respectively, is the prime catalyst delivering new types of services to society. In the recent few years, the area of wireless communications has witnessed a remarkable progression that has completely revolutionized the way we attend our daily activities. Recently, with the advent of $5^{\text {th }}$ Generation (5G) of wireless networks, a huge proliferation in the scale and types of network devices, products, services, applications, and businesses is anticipated
[1]. This tremendous expansion provided by $5 \mathrm{G}$ is envisioned to play a vital role in the advancement of society and in the expansion of the world economy. The revolutionary $5 \mathrm{G}$ technologies are predicted to directly contribute about $\$ 2.2$ trillion to the global economy in a decade or so [2]. To bolster this economic revolution, a paradigm shift in the consumer electronics (CE) industry for beyond 5G (B5G) and $6^{\text {th }}$ Generation $(6 \mathrm{G})$ wireless eras is imminent.

Rapid evolution and revolution in the CE industry have been witnessed in the recent years. However, the CE industry is currently in a rough transition period where effective design, installation, utilization, and predictive-maintenance of electronics devices at a massively large scale in the B5G wireless era is forecasted to emerge as an immense challenge. 
The natural performance boundaries of the existing technologies compared to the developing new diverse and contradicting performance and design requirements for the future $\mathrm{CE}$ plead the necessity of a tremendous leap forward in the CE framework. Such stringent requirements in next-generation $\mathrm{CE}$ include context-awareness, light-weighted devices, communications ability with lower overhead, intelligence provision, automation, security and privacy provisions, high energy-efficiency, low-cost, holographic, remote-interaction, haptic interaction, assurance of low human electromagnetic radiations exposure, multi-connectivity, ultra-high reliability, ultra-low latency, massive access - to name a few. Along with these rigorous requirements, ensuring barrier-free (accessible or universal) design of products, services, and environments is another prime challenge for the CE industry. Furthermore, the rapidly increasing demands of computational, storage, and communication abilities with the assurance of low-cost and high energy-efficiency of the devices is a challenging factor that needs a new framework to deliver the future quality-of-life-experience demands of the societies, which is the main focus of this paper.

The potential of cloud services to support the growing demands of seamless computing and storage requirements of $\mathrm{CE}$ devices has been highly acknowledged [3]. However, the high network latency in service delivery has been the primary limitation in exploiting its full potential. Now, with the advent of the ultra-reliable low-latency communications (URLLC) feature in $5 \mathrm{G}$, offering $10^{-9}$ packet-error-rate (PER) with $1 \mathrm{~ms}$ end-to-end (E2E) latency, this constraint is significantly resolved. Further evolution in URLLC towards enhancedURLLC (e-URLLC) is anticipated in B5G/6G (in a decade or so) to realize $0.01 \mathrm{~ms}$ E2E latency with $99.99999 \%$ reliability $[4,5]$. This inclusive succession holds the potential to revolutionize the existing wireless $\mathrm{CE}$ framework through the provision of e-URLLC connectivity of the devices to the cloud and edge computing, storage, and other facilities. To this end, this paper motivates a novel framework for deviceand system-level innovation in next-generation CE. The main contributions of this paper can be highlighted as,

- The state-of-the-art of CE is reviewed where future device types, device design requirements, performance requirements, supporting 5G technologies (e.g., URLLC and edge computing), associated socioeconomic aspects, and vision for next-generation $\mathrm{CE}$ in the $6 \mathrm{G}$ wireless communication era context are highlighted.

- A novel edge computing-enabled e-URLLC framework for $\mathrm{CE}$ devices, named as edge computing for $\mathrm{CE}$ (ECCE), is proposed and its potential enabling techniques/tools are identified. The notion of "Computing as a service" supported through $5 \mathrm{G}$ wireless services is built. A low-cost, energy-efficient, and processor-less (i.e., minimal processing) device design is motivated.

- Open research topics and future recommendations for next-generation $\mathrm{CE}$ in the $6 \mathrm{G}$ wireless era are identified.

The rest of this paper is organized as follows. Sec. II discusses recent advancements in $\mathrm{CE}$ and provides an outlook of the next-generation CE requirements. In Sec. III, the proposed ECCE framework is presented. Sec. IV identifies various enabling techniques for the proposed framework and discusses the open research topics along with interesting future recommendations for next-generation $\mathrm{CE}$ in the $6 \mathrm{G}$ wireless era.

\section{RECENT ADVANCES IN CONSUMER ELECTRONICS (CE)}

Towards enabling e-URLLC, ultra-low energy consumption, low devices cost, and high capacity links targeted by B5G communications networks, in addition to several ongoing innovations at the network and system level, it is crucial to introduce innovation at the device-level by addressing the existing primary constraints such as power limitations, low computational capacity, and limited storage capacity of the devices, etc. In this regard, some of the potential innovations in CE include the provision of "edge processing as a service", inter-chip communications via $\mathrm{THz}$ line-of-sight links, novel interfaces to support e-URLLC links, lightweight modulation for high-speed communications, near-field communications, visual internet-of-things (IoT), blockchain, and backscatter communications for zero-energy devices, etc. This section mainly discusses the recent trends and advances in CE.

\section{A. RECENT TRENDS - WIRELESS NETWORKS AND DEVICES}

The standardization of $5 \mathrm{G}$ wireless networks commenced with the Release- 15 of $3^{\text {rd }}$ generation partnership project (3GPP) [6]. 5G has taken a tremendous leap forward by offering various innovative technologies and services. The primary $5 \mathrm{G}$ services are enhanced mobile broadband (eMBB), ultra-reliable low latency communications (URLLC), massive machine-type communications (mMTC), and tactile internet (TI) [7]. The 5G New Radio (NR) is expected to evolve in the upcoming releases of 3GPP. The number of mobile phone users around the globe today are estimated to be about $4.8 \mathrm{bn}$, where $3.5 \mathrm{bn}$ are smartphone users that are expected to increase to about 3.8 bn by 2021 . The average daily time spent for data/internet usage per smartphone is approximately $2 \mathrm{hr}$ 55mins, where the average data consumption is about 1.7 $\mathrm{GB} /$ month which is expected to increase to $8.9 \mathrm{~GB} /$ month by 2021. In the UK alone, there are about 60 million mobile users which consumed 6bn and 82bn calling minutes and messages (i.e., SMS/MMS) in 2017, respectively. The share of data/internet transmissions in electricity usage intensity was estimated as $0.06 \mathrm{kWh} / \mathrm{GB}$ in 2015 [8], and the $\mathrm{CO} 2$ emissions count was estimated about $0.277 \mathrm{kge} / \mathrm{kWh}$. Hence, for the UK alone, the data/internet contributes about 19,068 tonnes of $\mathrm{CO}$. Alongside, efficient E-waste management is another global challenge. Nevertheless, there is an immense need for smart proposals for shaping the next generation of electronics to meet the increasingly rigorous demands of future wireless networks along with the assurance of environmental protection. 
In the B5G/6G wireless era, by the year 2030, the global mobile and machine type subscribers are predicted to rise to about 17.1bn and 97bn [9], respectively. Various new types of devices are expected to emerge, e.g., wearable, body implants, textile embedded, human-machine interaction, smart cars, haptic sensors, smart home/office, network edge, consumer cognitive systems, and robots, etc. In a massively connected world of the future, a huge amount of data will be generated and communicated across device-, edge-, and network-levels of the communication skeleton. A $55 \%$ annual increase in mobile data traffic is forecasted for the years from 2020 to 2030 [9]. This predicted voluminous amount of data generation can be effectively utilized for creating new services, improving the grade-of-service (GoS), and raising the value generation opportunities provided that a matching device-, edge-, and network-level innovative design in privileged. In the provision of "access to information at the fingertips", meeting the insatiable processing-power demands at the device-, edge-, and network-sides requires a significant enhancement in the computing framework. With the anticipated massive increase in the number of devices, an increase in the chore of managing data across multiple heterogeneous devices (i.e., synchronization of contents) for the users will also arise. Moreover, the provision of high processing and storage capability for the devices with high energy efficiency and low cost is a prime research challenge for the $\mathrm{B} 5 \mathrm{G} / 6 \mathrm{G}$ wireless era. The advent of $5 \mathrm{G}$ technologies has extended new opportunities to rethink the existing computational and storage models as well as realize new business opportunities. The provisions of information exchange with ultra-low latency and ultra-high reliability (i.e., $10^{-9}$ PER with 1ms E2E latency $[4,7])$ and the advent of the architectural concept of edge computing in $5 \mathrm{G}$, besides supporting many dynamic applications, open new doors of opportunities for rethinking the device- and system-level architectures in next-generation CE.

\section{B. DEVICE TYPES - FUTURE PROSPECTS}

Humans in the B5G digital society are expected to see enormous advancements in the types and quantity of network devices for augmenting the effective creation, processing, exchange, storage, and utilization of information. The use of smartphones, tablets, and computers may continue with some added features, while a boom in the device types for B5G/6G wireless networks is anticipated. The mMTC services of $5 \mathrm{G}$ are expected to revolutionize various areas of the future World through extending the provisions of sensing, communicating, and actuating in machine-type devices, e.g., the smart-industries, -cities, -homes/offices, healthcare, -agriculture, -mobility, etc. Furthermore, various context-aware and wearable devices for applications and services ranging from healthcare to seamless humanmachine interaction are expected to emerge. For example, such devices may include bio-nano in-body devices, brain sensors, earpieces, textile embedded devices, surveillance and security devices, vehicular devices $(\geq 100 \mathrm{~km} / \mathrm{h})$, etc.
The augmentation in machine learning (ML) and wireless communication methods combined with a plethora of such devices has the potential to signify intuitive and efficient interaction between humans and machines. Considering the nature, requirements, and constraints of such devices, it may not be attainable to furnish such devices with substantial battery and computing capabilities. In this context, offloading of the devices' computing tasks to the available computing resources at the network edge and/or network cloud through fast, reliable, and energy-efficient communication links is an attractive proposal to support the progression.

\section{HYBRID 5G CAPABILITY FOR DEVICES}

The network services requirements vastly vary over various diverse application scenarios, e.g., high data-rate may be required with high delay tolerance in broadband services while there is high-reliability and low-latency required in mission-critical IoT. Innovation at device-level providing the hybrid 5G services capabilities to the user devices (e.g., devices equipped with URLLC and eMBB services capabilities) can help in aggressively benefiting from edge computing platforms. In this context, the overall quality-of-service (QoS) may be defined by joint consideration of devices' energy consumption, network resources utilization, devices processing tasks' offloading rate, and network services' successful delivery. Such innovation at the device-level fully utilizing the edge computing platform can also potentially lead to a complete rethinking of the design of the devices, e.g., the induction of processor-less devices (with minimal processing capability). The provisions of edge computing, URLLCs, high data-rate communications, data generation and profiling, and location-awareness has a strong potential for creating new business opportunities for the (access) network operators for monetization of network services.

\section{ENHANCED-URLLC (E-URLLC) FOR CE}

The URLLC is one of the primary services offered by $5 \mathrm{G}$ which lays the foundation of advancements in delay stringent communication applications. This 5G service-type promises the reliability of $10^{-9}$ PER with E2E latency of $1 \mathrm{~ms}$, and it aims at supporting various mission-critical future application scenarios, such as autonomous cars, tele-surgeries, virtual/augmented reality, industry automation, machine-tomachine communications, smart grids, remote control in tactile internet (i.e., teleoperation systems, wireless virtual reality (VR), wireless augmented reality) - to name a few. The link reliability can be enhanced by consuming more channel resources while improving E2E delay has various diverse factors involved which require fastidious considerations. In 5G NR, fast grant schemes along with short frame structure are proposed to enhance the delay involved in access grant and air-interface, respectively. For example, the reference and control signals can be structured as transmitted in the beginning (i.e., "front loaded"), avoiding the use of time-domain interleaving in orthogonal frequency-division multiplexing (OFDM), etc, are among the contributing factors. More- 
over, very tight processing time requirements for devices (and network equipment) is another critical requirement. For example, the devices are required to respond with hybridautomatic repeat-request (ARQ) acknowledgement after receiving of the request within a time span less than that of a single slot (i.e., "mini slot" transmission). Moreover, the design of higher layers protocol stack is also critical. For example, the provision of appropriate selection of the header structure to enable the data processing without knowing the total amount of data planned to be transmitted can potentially support in enhancing the latency. Moreover, mobile edge computing (MEC) framework also significantly contributes in enhancing the URLLC experience (it also supports 4G devices). Nevertheless, still there exists significant scope to further improve the users E2E delay experience by exploring different other factors, particularly in the core network. Enhancing the devices design, system architecture, resources consumption efficiency, frame structure and protocols design, and network state prediction accuracy has a vast potential in enhancing the overall URLLC services in B5G era. To this end, an evolution of URLLC towards e-URLLC in B5G/6G era is anticipated to deliver $0.01 \mathrm{~ms}$ E2E latency with $99.99999 \%$ reliability $[4,5]$.

\section{E. MOBILE EDGE COMPUTING (MEC)}

MEC extends the provision of computational capabilities available at the edge of the mobile network. The availability of computing (and storage capabilities) in close proximity of the users has a strong potential in enhancing the network services through locally meeting the users' demands. In Release 15 of 3GPP, support for edge computing has been defined. The user plane function (UPF) located closer to the devices/users extends the support for data traffic services, i.e., a gateway that is closer to the edge serving station is selected. Ensuring the routing of the traffic through a local gateway instead of a gateway located in the core network provides benefits in terms of reduced E2E latency and transport bandwidth requirements. 3GPP is advancing towards enhancing the MEC architecture for improving edge applications, see e.g., [10]. In this context, architectural advancements for improving configuration, discovery, selection, connectivity, registry, mobility management, and hosting third-party services, etc, are the prime research directions.

Intelligent caching of the popular contents at the edge of mobile network, i.e., within the radio access network (RAN), can significantly reduce latency and boost the data-rate for all types of users (even the users not using the latest air-interface technologies). Besides the revolutionary air-interface technologies offered by $5 \mathrm{G}$, more extensive software-defined approaches to network virtualization are also leveraged. In this context, MEC is a key 5G technology which with its support for network programmability delivers the demanding requirements of network scalability, throughput, latency, and automation.

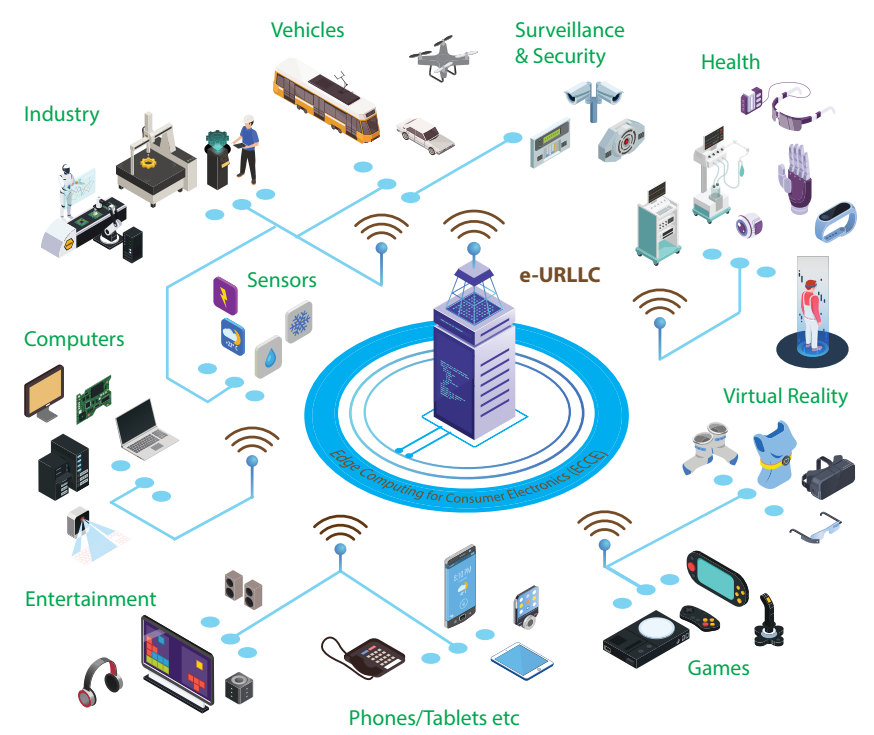

Figure 1: Example $\mathrm{CE}$ device types and edge computing platform (i.e., ECCE).

\section{F. IOT EDGE COMPUTING (IEC)}

In today's world, access to information and its exchange is at the fingertips. One of the next paradigms at the forefront is IoT services, which aim at connecting physical things with the internet to exchange the sensed/observed information. Most of the standard IoT networks are supported through cloud-computing platforms. Now the consumer IoT has become a multidisciplinary ecosystem that has begun to expand very rapidly, e.g., expansion in the use-cases of homes, offices, cities, energy grid, defense, health, agriculture, education, transportation, etc. The massive amount of data sensed and generated from environmental, healthcare, and other applications require matching processing capabilities to best benefit from it at the device and network levels. In various scenarios, the demands of real-time data processing and feedback require low latency connectivity to the processing/computing facilities, e.g., for the scenario of connected autonomous vehicles, etc. To this end, since the IoT devices cannot be provisioned with heavy processing and storage capability, devising a robust IoT edge computing (IEC) platform to support such IoT devices is a promising solution [11]. Furthermore, another associated open research challenge for increasingly pervading IoT networks is the assurance of security and privacy provisions, as the IoT devices are generally not designed with security and privacy features [12]. Innovation at device-level and edge computing platforms is required to ensure security and privacy provisions.

\section{G. SOCIOECONOMIC ASPECTS OF CE}

Today, CE products are more than just technology innovations and have become a vital influencing player in the ecosystem of socioeconomics. This marks the electronic engineers as the architects of tomorrow with the responsibility 
to address various dynamic challenges even beyond the realm of science and engineering. The future wireless networks are expected to play a vital role in the socioeconomic ecosystem [1]. Communication networks are becoming a central platform for daily life services delivery, e.g., mobile applications for Taxi services, food delivery, online shopping, etc. Eventually, when all (most of) the daily life services will be delivered through communication networks platform, the performance of wireless links will directly influence the overall grade of service delivery. In a massively connected world of tomorrow, the socioeconomic aspects respond at a very rapid pace and on a global scale. The revenue generation rate is directly influenced by the provided grade of service (GoS), where response time to the services call is critical. The consumers' perception of the service quality is also very critical in the success of business in the current business model. Therefore, the quality-of-experience of the services by the consumers is required to be more comprehensively quantified with a holistic view of the impact and performance of each aspect, e.g., capturing the user perception, system performance, and context, etc. Furthermore, in such massively connected and densely populated networks, the amount of data generated across the network can be utilized for network performance improvement as well as for value generation [1]. However, provision of data privacy and security is emerging as one of the prime concerns of the future networks.

\section{H. DEVICE-LEVEL INTELLIGENCE FOR CE}

5G has laid the foundation of AI provisions in network operations through some isolated AI operations. The anticipated expansion in these provisions yields the potential to achieve fully-intelligent network orchestration and management in the $\mathrm{B} 5 \mathrm{G} / 6 \mathrm{G}$ era. Along with the core-network operations, the scope of ML and deep learning (DL) for device-level, air-interface, and network edge is also well recognized. The limited battery, processing, and storage capabilities of the devices limit the scope of employing learning algorithms at the device-level. However, if provided with the learning capabilities, the CE devices can contribute significantly to boost the overall system performance as well as to derive new types of services. The highly serviceable information available at the device-level, such as location, channel, mobility, healthcare, battery life, etc, motivates the investigation of employing data-driven methods at the device-level. To this end, the devices connected to an edge computing platform through wireless links provide an imminent solution in the realization of the learning potential of device-level data. In this regard, the deployment of emerging learning paradigms, such as ML, DL, Quantum ML (QML) - to name a few, at the network edge to assist device-level operations can be considered a potential research direction for the $\mathrm{B} 5 \mathrm{G} / 6 \mathrm{G}$ era.

\section{PROPOSED EDGE COMPUTING FRAMEWORK FOR NEXT-GENERATION CONSUMER ELECTRONICS}

The URLLC and edge computing features of $5 \mathrm{G}$ provide the industry and research communities with the privilege to rethink innovative use-cases for realizing their full potential. The future generation of wireless networks (i.e., B5G/6G) is expected to support significantly high-capacity and lowlatency short-range communications. To this end, it can be envisaged that future $\mathrm{CE}$ will be at the forefront of innovation to consume the offered wireless capabilities in the B5G/6G era. Considering the contemporary latency requirements of CE (e.g., entertainment, gaming, etc.), to enhance the commercial potential of "processing as a service", the research community intends to commit to the evolution of URLLC towards e-URLLC in the $\mathrm{B} 5 \mathrm{G} / 6 \mathrm{G}$ era. This succession is determined to achieve a reliability of $99.99999 \%$ with $0.01 \mathrm{~ms}$ E2E latency in a decade or so [4]. Such ultra-high reliability and ultra-low latency abilities can amply facilitate the device-design in terms of device-cost to support a wide range of $\mathrm{CE}$ devices. The processing capabilities available at the edge can be provisioned to the $\mathrm{CE}$ devices to facilitate them as processor-less (or minimal-processor) design. The devices/users can rent the most expensive part "the computational processor" as an "edge processing service" on a "pay as you go" basis through a real-time on-demand eURLLC wireless connectivity. Appropriate modeling of such a demand and device-design is vital to serve a wide range of processing units and latency demands.

The basic concept of the proposed ECCE framework to deliver on-demand computing services to $\mathrm{CE}$ devices through e-URLLC wireless connectivity is illustrated in Fig. 1, where different $\mathrm{CE}$ device types are also shown. The latency involved in offloading of computing tasks from the CE devices to an edge computing platform is anticipated to become a matching case to that of performing the tasks locally at the devices along with the anticipated evolution in link-speed, reliability, and -latency performance in the B5G/6G era. The associated potential innovations include: 1) replacing cabling requirement between the computational processor and corresponding user interfaces (e.g., between CPU and Monitor, etc), 2) inter-chip communication within CE circuitry via THz line-of-site links, and 3) processor-less devices (minimal processor) supported through the proposed ECCE platform.

The proposed ECCE platform can be hosted at the network access points (e.g., base station (BS), evolved node B (eNB), radio network controller (RNC), wireless fidelity (WiFi) routers, etc). The architecture of such access points and key device-level design features are shown in Fig. 2. The employed Services Classifier at the access points classifies and diverts the traffic/data received from the $5 \mathrm{G} / \mathrm{B} 5 \mathrm{G} / 6 \mathrm{G}$ radio interface to an appropriate forum, e.g., ECCE, cloud services, or internet platforms, without any loss in performance for network data users. For example, this can be achieved through bundling the traffic/data with the operation that is requested on that data (i.e., encapsulation of packets). Afterward, the respective service manager handles the subsequent 


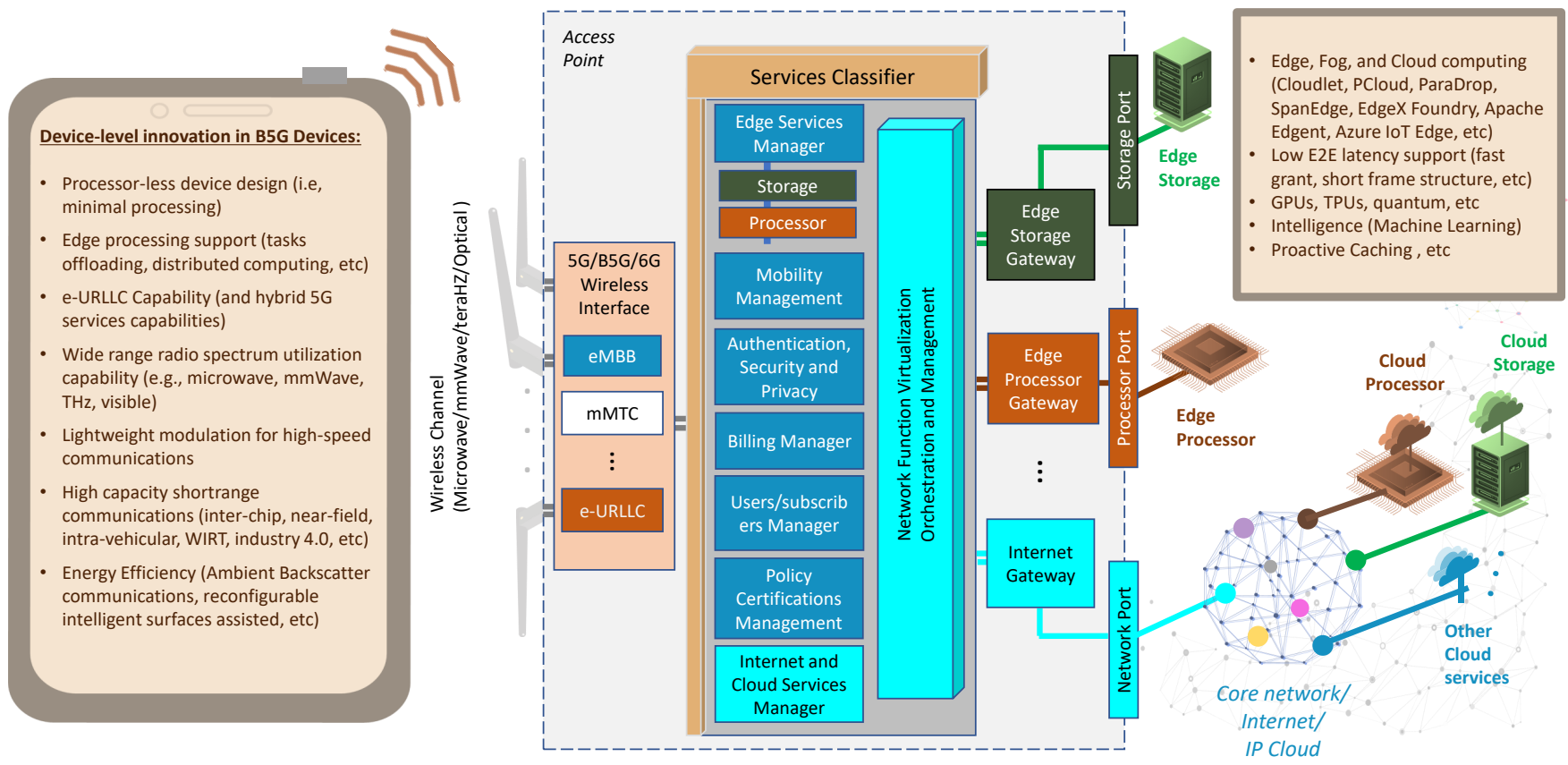

Figure 2: Access point architecture for hosting the proposed ECCE platform, with the indication of some key device- and system-level features.

operations; e.g., the Edge Services Manager (see Fig. 2) manages the edge services (computing, storage, etc) operations. A gateway provides access to each service type through the respective port/interface, e.g., Edge Storage Gateway, Edge processor Gateway, Internet Gateway, etc. Given the robust processing capability available at the network edge (e.g., at access points), along with enhancing the network experience for the mobile users it can also support the CE devices in their local processing/computational tasks. The processing-time of the computational tasks of the $\mathrm{CE}$ devices can be reduced through their processing at a powerful processor interfaced at the network edge, however, the latency of wireless links from the CE devices to the edge platform is critical in enhancing the overall performance tradeoff. Cloud processing facilities (deployed in the core network), on the other hand, may not be suitable for such customary local processing tasks due to the high latency involved in the backhaul and core network. Mobility management, authentication, security, privacy, policy certification, subscription, billing, operations can be looked after by the respective operational blocks, as indicated in Fig. 2.

The proposed ECCE framework also holds the potential to deliver improvement in devices' energy efficiency performance (to enhance their battery life). This ultimately aids in building an environment friendly CE eco-system to help the climate. For the devices relieved from the burden of performing heavy computational tasks locally, the energy efficiency trade-off between the energy saved at the device-level in the account of reduced computational tasks and the energy consumed on communicating the tasks to the ECCE platform through e-URLLC links is critical. Deploying multiple ECCE platforms and ensuring appropriate association of CE devices with the platforms can help in further enhancing the overall latency and energy efficiency. The window of opportunity provisioned by the proposed ECCE platform in terms of optimizing the overall energy consumption, device cost, and $\mathrm{CO} 2$ emission count trade-off is illustrated in Fig. 3. With the edge computing platform equipped with heavy processing capabilities, the device design can be provisioned with minimal processing capabilities resulting in significantly reduced device cost. For employing the proposed ECCE platform, the cost of the edge-side will increase, however, the cost per connected device (to ECCE) will reduce in the context of a massively connected network of the future (as the local processors in the devices usually stay in idle state). The device-level innovation required to exploit such a framework includes the provisions for hybrid 5G services, processor-less design (minimal processor), the ability to utilize a wide range of radio spectrum, etc. Rethinking of the access point design is required to add the support for connectivity for external processors through parallel ports/slots where processors of different capabilities can be plugged in. For such accesspoints/network-edge design, an appropriate external processor for a certain use-case can be installed, e.g., a heavy-duty processor for the laboratories to handle the tasks of all the connected wireless devices, etc. Furthermore, such an edge computing framework can also facilitate the $\mathrm{CE}$ devices to benefit from various emerging revolutionary processing and learning technologies, e.g., quantum computing, quantum ML, etc, through the integration of such facilities at the edge.

The proposed ECCE framework also opens vast new business (value generation) opportunities for the network (ac- 


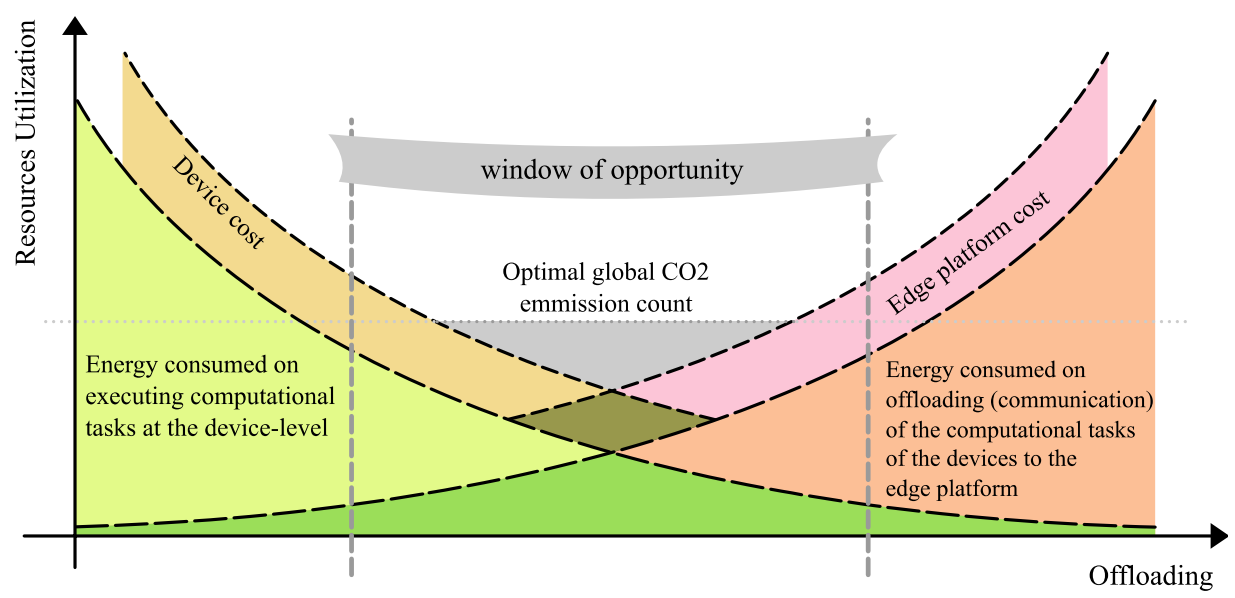

Figure 3: Window of opportunity for improving performance trade-off between devices' energy efficiency and cost facilitated through the proposed edge processing platform.

cess) operators through the lending of on-demand processing services to the wirelessly connected CE devices. Such a service can be termed as "processing as a service" and it has immense potential in facilitating device-level innovation. The use cases for "processing as a service" concept are illustrated in Fig. 4. For example, the access point in a coffee-shop/cafe is equipped with a heavy-duty processor, which extends on-demand computing service to the user devices through wireless e-URLLC links. The service may be purchased by the users for a certain time duration or a certain amount of computational tasks as prepaid cards or codes from the cafe reception. Similarly, in research laboratories, libraries, hospitals, offices, homes, and public waiting areas, such ondemand services can be provided to the devices to offload their computational tasks. It can be, thus, envisaged that edge-computing together with e-URLLC for CE devices, besides supporting mission-critical applications, can facilitate various new-types of services to the network subscribers, enterprise users, and allied industries in the $\mathrm{B} 5 \mathrm{G} / 6 \mathrm{G}$ era.

\section{ENABLING TECHNIQUES/TOOLS AND FUTURE RECOMMENDATIONS}

The key enabling technologies/tools to exploit the full potential of the proposed ECCE framework are discussed in this section. Besides, some open research problems and future recommendations are also highlighted. In Fig. 5, a taxonomy of the key enablers and proposed innovations at the deviceand system-levels is presented. The need for a new business model for network edge operators in order to serve the motivated notion of "processing as a service" is also indicated.

\section{A. EDGE COMPUTING AS A SERVICE}

The current trend in analyzing the massive amount of data from IoT devices is towards IoT-cloud convergence, in which a centralized cloud server processes the huge amount of IoT/sensor data and stores for future usage. Although, cloud computing is an excellent platform to handle and store the huge amount of data generated from the IoT systems, this is not suitable for applications demanding real-time operation and high Quality of Service (QoS) requirements [13]. To address this issue, edge computing, also called fog computing, is considered an important enabler due to its several benefits [13,14], including the following: (i) Proximity: Processing closer to the devices (at the BSs/eNodeBs or aggregation points), (ii) Lower Latency: considerable latency reduction is possible, (iii) Location awareness and real-time access to radio network and context information, (iv) high bandwidth availability at the edge and reduction of backhaul bandwidth. Various edge computing/processing and storage nodes can be distributed across the network, with the IoT devices/endusers on one side and Cloud-center on the other side.

\section{1) Use-cases and implementation tools}

Edge processing finds applications in various practical scenarios including industrial automation, remote monitoring in industry, autonomous vehicles, augmented reality (AR) and VR, Tele-medicine, connected homes and offices, smart farming and agriculture, predictive maintenance, video monitoring, blockchain and smart cities/homes. In the following, we highlight various use-case applications of edge processing along with some examples in the aforementioned scenarios.

1) Use Case 1: IoT Traffic/task Offloading: (i) From sensors/actuators to the edge-server, (ii) From the edgeserver to the cloud-server, (iii) From the cloud-server to the edge-servers, and (iv) From the edge-server to sensors/actuators

2) Use Case 2: Decentralized network operation/orchestration:

(i) Autonmatic functioning of edge nodes in case of unavailability/failure of backhaul links (network resilience), (ii) Edge caching to deliver contents closer to the users, and (iii) data aggregation at the edgeaggregators

3) Use Case 3: Formation of localized networks: (i) Support for localized services (i.e, adverts, traffic informa- 


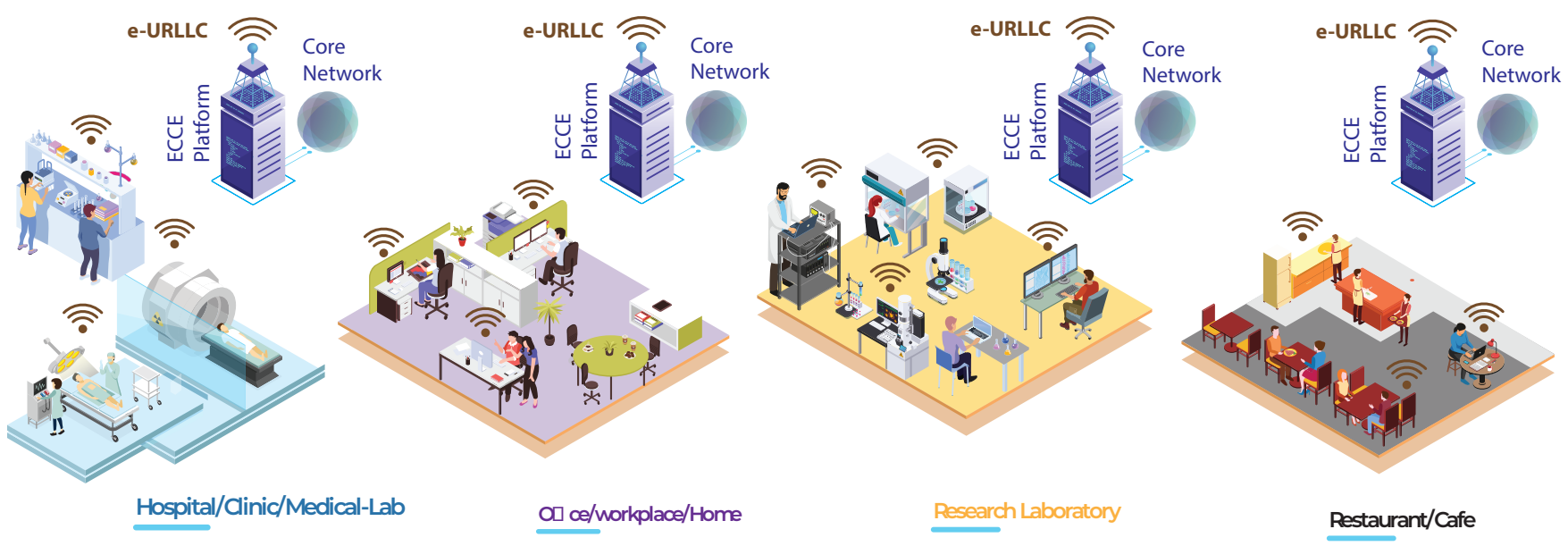

Figure 4: Illustration of some examples scenarios/use-cases of the proposed ECCE platform.

tion, etc.), (ii) location-aware resource allocation by considering dynamically varying patterns (i.e, service demand, mobility etc.)

4) Use Case 4: Factory and enterprise communication: (i) Support for industrial and enterprise IoT, (ii) Ultralow Latency and ultra-high reliability, and (iii) Secured connection to external networks

Various tools are available to realize and deploy the concept of edge/fog computing including FogNetSim++, iFogSim, FogTorchII, EdgeCloudSim, IoTSim, EmuFog, Fogbed, NS-2/NS-3, Graphics Processing Unit (GPU), High Performance Computing (HPC) and Software Defined Radio (SDR). In order to connect edge-devices and process data at the edge, various IoT platforms including Arkessa, ThingSquare and ThingWorx exist. Similarly, various lightweight messaging protocols such as constrained application protocol (CoAP) and messaging queue telemetry transport (MQTT) have been implemented in order to effectively collect and process the data from IoT connected devices [15].

\section{2) Edge Processing from Business Perspectives}

The widely followed business model in the cloud-based IoT data analytics is a high fee pay-as-you-go business model. This model can not satisfy real-time requirements of latencysensitive applications and also it creates data privacy concerns. This has led to the need of shifting data analytic functionalities to the edge of the network.

Various sensors and machine-type devices such as industrial machines, smart home equipments, medical devices, smart meters, built-in sensors in mobile phones, planes, cars, and body area sensors produce a massive amount of data to be processed in the real-time. However, the existing cloudcentric architecture adopts distributed streaming architecture to collect and process the data at the cloud, and is not suitable to meet real-time constraints of latency-sensitive IoT applications. Also, the "pay-as-you-go" business model adopted by this architecture is not suitable for the end-users from the cost and ever-increasing data-volume perspective. Furthermore, the current cloud-centric architecture is not suitable to maintain the data privacy of local communities and people [15].

Moreover, existing edge analytic techniques are not flexible to support the domain-specific processing of edge IoT applications. Also, resource utilization efficiency is crucial to be considered due to limited computational and storage resources at the edge side.

\section{B. DEVICE-LEVEL ENERGY EFFICIENCY ENHANCEMENT}

This section discusses promising approaches for enhancing device energy efficiency in $6 \mathrm{G}$ wireless networks.

\section{1) Advanced energy saving features}

Minimizing energy consumption at the devices and edgeservers is crucial for enhancing the energy efficiency of $6 \mathrm{G}$ wireless networks. In one hand, due to several components involved in smart devices such as operating systems, apps and screen display consume a significant amount of the available energy budget at the device-side. On the other hand, resourceconstrained nature of low-end sensors and IoT devices are not able to employ energy-hungry or computationally intensive algorithms at the device-side, leading to the need of energy saving mechanisms at the device-side. In this regard, there are already some energy saving mechanisms at the deviceside being incorporated for $5 \mathrm{G} \mathrm{NR}$ as compared to the conventional long-term evolution (LTE) devices.

The energy consumed by a device per transmitted bit in a $5 \mathrm{G} \mathrm{NR}$, in general, is minimized by enabling the higher data rates and lower latency as this facilitates to terminate the data sessions faster than in the LTE case. Nevertheless, due to several new features included in 5G NR such as shorter time slots, wider bandwidths and multiple scheduling events per time slot, the energy consumption at the device-level increases, and to address this, 3GPP Rel-15 of the NR specification has proposed various energy saving mechanisms 


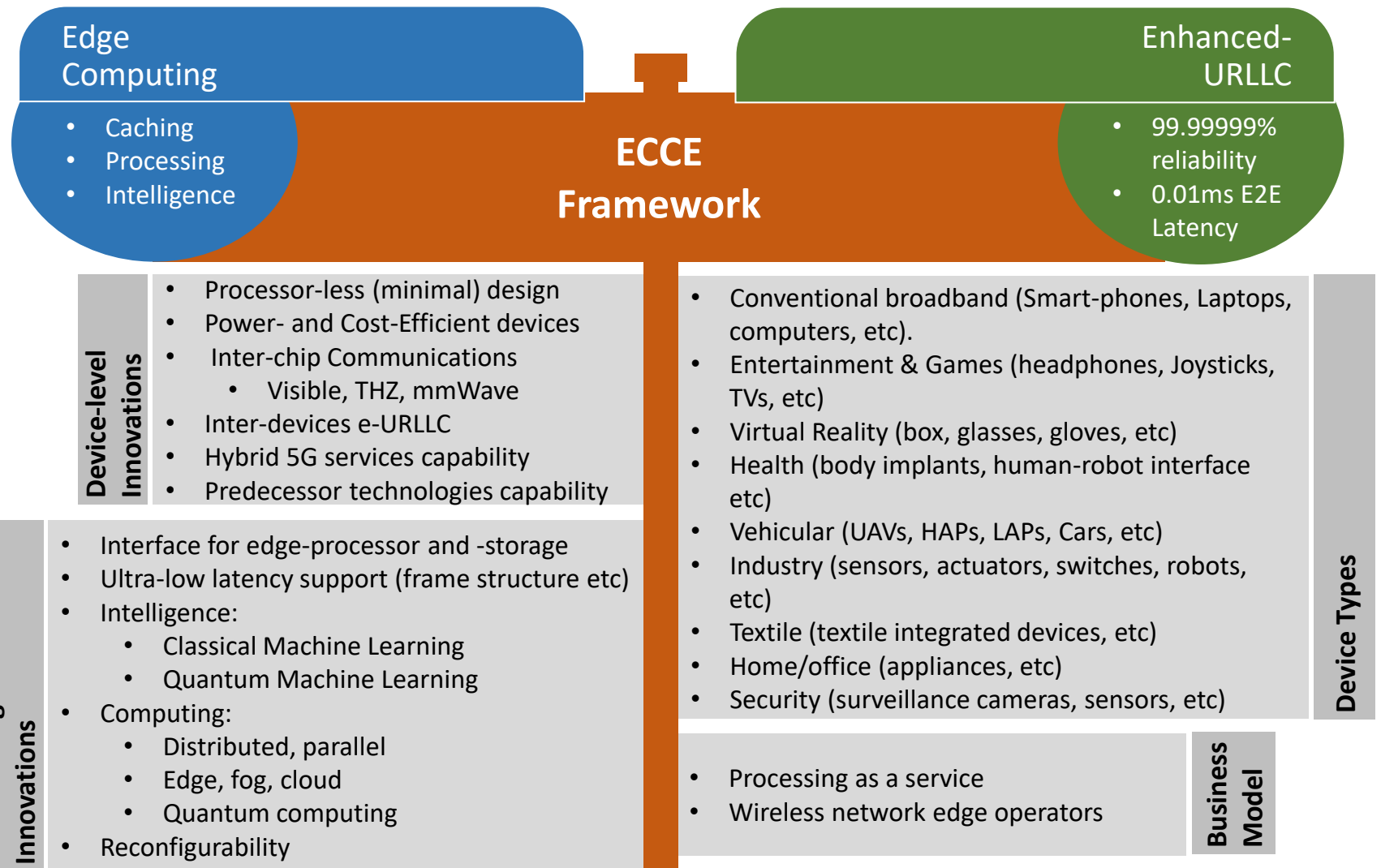

\section{B5G/6G Wireless Era}

Figure 5: The proposed e-URLLC supported edge computing for wireless consumer electronics (ECCE) framework for deviceand system-level innovation in B5G/6G wireless era.

such as customized control channel monitoring, connectedmode Discontinuous Reception (cDRX) and inactive state. Furthermore, several additional features are being included for device energy savings in 3GPP Rel-16, including the following: (i) dynamic cross-slot scheduling, (ii) dynamic SS adaptation, (iii) connected-mode wake-up signal, (iv) secondary cell dormancy and (v) device assistance for secondary cell release. By configuring the cDRX and Search Space (SS) parameters, network vendors can balance the trade-off between device energy efficiency and system performance metrics such as latency and throughput [Ericsson].

\section{2) Ambient Backscatter communications}

Backscatter Communications (BsC) can enable IoT devices to modulate an incident RF signal (instead of a locally generated new carrier) and reflect back to the receiver in an overthe-air modulation fashion [16]. Furthermore, ambient BsC is a promising solution towards providing continuous power supply to massive IoT devices due to its high power efficiency and spectrum efficiency. It has a huge potential to address the issues of limited battery life, power and spectrum resources cost, and also to avoid the tedious tasks of recharging, replacing and maintaining the batteries in battery-operated
IoT devices/sensors, by enabling the deployment of batteryless passive devices in massive-IoT networks. Also, due to low manufacturing cost of backscatter nodes, BsC can significantly reduce the deployment and operational costs of massive IoT scenarios such as logistics and supply chain management and industrial automation.

Despite the huge potential of ambient $\mathrm{BsC}$, there are several challenges to be addressed. First, as the transmission efficiency of BsC depends on the ambient RF source, the ambient $\mathrm{BsC}$ needs to be designed for a particular ambient source. Second, the scheduling of transmission data for backscatter devices becomes challenging due to the dynamics of ambient signals. Third, ambient $\mathrm{BsC}$ design should guarantee the interference protection of licensed users while utilizing ambient signals from the licensed sources. Fourth, accurate resolution of simultaneous backscatter signals received from multiple illuminated $\mathrm{BsC}$ devices demand for appropriate multiple access mechanisms.

\section{3) Reconfigurable Intelligent surfaces}

Existing wireless systems are based on the principle of fixed radio environments without having any configurable/control features, and combatting the harmful effects of the radio 
environments has been one of the main objectives of the most existing protocols/algorithms. One recent revolution in this regard is to utilize electromagnetically active surfaces, i.e., Reconfigurable Intelligent Surfaces (RISs), towards enabling a programmable and controllable smart radio environment, which itself can be used as a degree of freedom for optimizing $6 \mathrm{G}$ wireless systems. By artificially re-configuring the radio propagation environment of electromagnetic waves, such smart radio environments can be useful to significantly reduce energy consumption and enhance the spectral efficiency of future wireless networks. Recent works in the domain of RISs have shown that these intelligent surfaces can effectively control various parameters of the incident electromagnetic waves (i.e, amplitude, frequency, phase, polarization) without the need of any complex encoding, decoding and RF signal processing operations [17]. As compared to the conventional passive meta-surfaces with fixed electromagnetic functionalities, RISs should be re-configurable in order to adapt the parameters of the incoming waves with regard to the variations in dynamic wireless environments. As the modeling, design and analysis of RISs involve different interdisciplinary fields including physics, electromagnetic theory, communication theory and wireless communications, current meta-materials should be redesigned by incorporating the features of communications engineering while considering the interactions between digital and physical worlds.

In emerging B5G/6G IoT networks, the incorporation of RISs along with the backscatter communications can significantly reduce the amount of energy required to report the sensed data by the IoT sensors to the fusion centers/edge servers. By realizing the IoT devices with RISs and by utilizing the concept of backscattering, the sensed data can be piggybacked into the shape of the reflected signals without any overhead and energy cost [17]. Employing RISs also has a strong potential in boosting the converge and performance of UAVs-assisted future wireless networks [18]. Furthermore, RISs can significantly assist in enhancing the security of wireless links at the physical layer of protocol stack. In [19], a secrecy performance analysis of an RISassisted wireless communication system is analysed in the presence of an eavesdropper, where the potential of RIS in enhancing the secrecy performance in demonstrated.

\section{SECURITY FOR WIRELESS CONSUMER DEVICES}

With the advent of $5 \mathrm{G}$ services influencing almost every walk of life, an extensive expansion in the number of wirelessly connected consumer devices is anticipated globally [16]. The assurance of security and privacy provisions in such massively connected wireless networks has become extremely essential to counter any intrusions, data privacy breaches, cyber-attacks, etc. In the context of the proposed framework, the computational tasks offloading from consumer devices to the edge-computing platform raise new concerns of security and privacy. Guaranteeing the security demands of such wireless consumer devices requires devising new innovative solutions. In this context, the potential technologies and research directions include physical layer security [20,21], classic and quantum cryptography [7], blockchain [22], AI-driven [23] techniques - to name a few. Developing novel security and privacy quantification metrics (security capacity) that thoroughly apprehend the potential risks and network state of future complex networks is another critical research direction $[24,25]$.

\section{HIGH CAPACITY SHORT RANGE COMMUNICATIONS}

Due to recent advances in cost-effective Complementary metal oxide semiconductor (CMOS) IC technologies, Terahertz $(\mathrm{THz})$ waves $(300 \mathrm{GHz}-3 \mathrm{THz})$ have recently got attention for a wide-range of daily-life applications ranging from 3D imaging and electronic smelling to space communications networks and very high data-rate $6 \mathrm{G}$ wireless communications. Towards enabling $\mathrm{THz}$ communications for $6 \mathrm{G}$ networks, research communities are investigating various aspects of the required software and hardware modules including $\mathrm{THz}$ channel estimation, $\mathrm{THz}$ beam tracking, $\mathrm{THz}$ modulators, $\mathrm{THz}$ solid state superheterodyne receiver and THz antennas. THz communications can be broadly categorized into: (i) Solid state $\mathrm{THz}$ wireless comm. system, which is based on frequency mixing mechanism, and (ii) Direct modulation $\mathrm{THz}$ wireless comm. system, which is based on the modulation of the baseband signals directly to the RF. Despite promising benefits of $\mathrm{THz}$ communications, there are several challenges such as developing THz RF devices due to the required non-linear modeling and parameter extraction, supporting the required modulation depth and speed for the THz modulators, need of compact planar patch antennas, and low-complexity signal processing in $\mathrm{THz}$ band.

The other prominent research directions include Industry 4.0 (no cabling requirements), $\mathrm{THz}$ communications for inter-chip communications [26], Near field communications and Micro-beamforming, and Intra-vehicle/-car/-avionics communications (short-range wireless asynchronous real time (WIRT)) [27]. However, for such communication links, there is a need for advancements in lightweight modulation schemes, e.g., improvement in data rate offered by noncoherent communications, etc.

\section{E. COMPUTATIONAL TASK AND DATA OFFLOADING}

To provide seamless and low latency services to the consumer and enterprise end-users for a wide variety of devices including mobile and IoT, wearable, VR, industrial robots, autonomous, factory and satellite vehicles, amount of computational tasks and data to be handled will be massive. The edge servers can be facilitated close to the end-users including access points, base stations, gateways, traffic aggregation points, etc. and they need to handle all necessary heavy computational and data analytic tasks. Several edge node facilities are proposed based on the location and hardware configurations, including the following: OCTEON Fusion Family on a small cell base station, Nokia's software solution in base stations, Cisco's IOx in service router and Intel's Smart Cell Platform [28]. 
Larger size computations and data analytical tasks would certainly degrade the network reliability and latency. Two explicit solutions are: (i) performing computations and data analytic operations in a distributed framework through the edge servers within a certain geographic location and (ii) offloading the heavy computations and massive data to redirect to the cloud servers for further operations and sending back to the end-users. However, computational tasks and data offloading from edge servers will create many challenges. Depending on the capabilities and locations of the edge servers in a distributed environment the automated scheduler will deploy all tasks sequentially and hierarchically. To achieve this, a software-defined network (SDN) can pose an achievable solution under a distributed environment with logical and centralized control along with many other solutions in near future.

\section{F. DISTRIBUTED COMPUTING FRAMEWORK}

To support large-scale computing demands of future applications, employing distributed computing frameworks is regarded as an intuitive solution scheme. It is one of the fundamental concepts that undergirds today's online services platforms. Cloud computing is a centralized computing framework and it has proven a less privileged platform for real-time applications, in terms of associated high E2E latency. A guaranteed low latency is obligatory in seamless offloading of the computational tasks, while in the cloud computing framework the services are stretched over multiple networks and distributed over large geographic regions which make it a less suitable framework for real-time applications. Computing operations are required to be performed much faster and closer to the source of data and application users to provide guaranteed seamless services. Under the $5 \mathrm{G}$ and beyond networks, edge devices will produce a large number of data streams and analytics queries, where real-time decisions are essential. Performing such operations in a distant cloud will degrade the services. Many latency-sensitive realtime applications require a latency of $\leq 50 \mathrm{~ms}$ response time such as devices for visual guidance, video streaming, online gaming, etc. [28]. In this regard, the distributed computing on edge servers, i.e., next to the application users, can potentially provide an efficient solution in such cases. Although the distributed computing framework has been researched for over three decades, more dedicated efforts are still required to develop software/algorithms/tools for core enabling technologies and service provisioning to build the distributed environments, define nodes, virtualize the operations, and ensure services orientations and provisions, etc.

\section{G. SYSTEMS, TOOLS AND MATHEMATICAL}

\section{MODELLING FOR EDGE COMPUTING OPERATIONS}

Computing infrastructure and edge application deployment play the central role for edge computing operations by integrating computing power, storage, and network resources. Widespread research has been carried out through the collaboration of academia and industries on its architecture, energy efficiency, computational power, etc., since the concept of edge computing was introduced. Based on the innovation in architecture, programming tools and applications, there are many edge computing systems and tools proposed so far, viz: Cloudlet, CloudPath, PCloud, ParaDrop, SpanEdge, CloudSea Computing Systems, Cachier and Precog, FocusStack, AirBox, Firework and open source projects: CORD (Central Office Re-architected as a Datacenter), Akraino Edge Stack, EdgeX Foundry, Apache Edgent, Azure IoT Edge [29]. Depending on the type of target devices, edge nodes, computational tools, each of these edge computing tools/systems vary in design and features.

Alongside the discussed developments in variety of developed systems and tools, the idea of edge computing is also evolving for decentralization of the cloud resources through the enhancement of edge capabilities. This can be supported through enabling of low-latency communications, provision of sustainable energy usage model, and advent of scalable smart computation techniques. Soft computing libraries, algorithms and mathematical models with moderate computing operations can be seen as the key mathematical framework for successful implementation of edge computing operations. Advanced mathematical models such as multidimensional graph, game-theoretic models, and optimization techniques along with state-of-the-art ML techniques such as deep learning, reinforcement learning, and ensemble learning are regarded as the potential enabling tools for edge computing intelligent operations.

\section{H. PROBABILISTIC COMPUTING}

Although deterministic computing is the core technique for modern devices including computers and IoT devices it has many practical limitations. Deterministic algorithms for higher complexity problems may have huge computational costs, and different hardware features may also degrade device performance $[30,31]$. With the recent advancements in probability theory and statistics for machine learning, probabilistic computing has shown great promise in several application domains to achieve high-quality solutions and the efficiency and accuracy of achieving those solutions. Probabilistic algorithms use random bits in addition to the input, and the output and performance of the algorithms may vary depending on random bits. The fundamental challenge is the potential mismatch between the traditional hardware configuration and the highly stochastic nature of probabilistic computing [30]. A significant performance gain is only possible when such algorithms are carefully implemented in conventional systems to match the requirement of probabilistic computing techniques. A potential way forward could be a transition to quantum computing systems, which uses the probabilistic rules of quantum physics. Quantum computing has shown great theoretical promises; however, there are many technical hurdles to build practical machines due to the requirement of sophisticated materials, and the extreme environment to run those machines, particularly, for daily use. Therefore, a solution could be to develop a probabilis- 
tic computing system with 'p-bits', which would require a simpler technology, and run at room-temperature [31]. The benefit of probabilistic computing is enormous, and the 'pbits' probabilistic computer system for daily use and 'q-bits' quantum computing would revolutionize the digital era if fully exploited.

\section{QUANTUM COMPUTING AND COMMUNICATIONS}

The quantum mechanics concepts are envisioned to revolutionize various sectors of daily life including computing and communications [7]. The CE devices built from elementary particles are subject to exercise the principles of quantum mechanics enduring a strong association with the postulates of Euclidean geometry. The fundamental concepts of quantum superposition, quantum entanglement, or the no-cloning theorem offer an immense amount of parallelism in the computing concepts, which has also received the endorsement through recent demonstrated results. A qubit can simultaneously hold both the binary states. The number of unique binary patterns that can be represented simultaneously with multiple interacting qubits is in the order of 2 , which is significantly higher than a single binary pattern at-once in classical boolean representation. Therefore, such computing systems can effectively generate counter-intuitive statistical data patterns that are beyond the reach of classical computing systems [7]. As recognition of these potentials, new disciplines like "Quantum Computer Science" and "Quantum Information Science" have also originated.

In the exploitation of these physical quantum mechanics phenomena, a revolution in communications and computing sectors is envisioned to be around the corner [7]. In the telecommunications sector, the advantages offered by quantum teleportation and cryptography concepts make quantumassisted communications superior to classical communications in various aspects (e.g., enhanced channel capacity and security, etc). In the computing sector, a target of realizing quantum computers with 100 and 1000 qubits was set in 2016 as short- and long-term goals of 5 and 10 years, respectively [7]. Subsequently, in 2018, Google announced a 72 qubit superconducting quantum computer [7]. Despite the rapid advancements being witnessed in quantum computers recently, still, a dedicated effort is required to make them operational at practical/normal temperatures. However, in the context of a quantum computing facility provisioned at the network cloud or edge, the requisite low-temperature requirements can be met. In the context of the proposed framework, with such tremendous computing capability employed at the network edge to support e-URLLC enabled wireless devices, a revolution at device-level and architecture-level design can be foreseen. Such a framework can not only translate the provisions of enormous computational power to the learning capability but it can also be seen as a fundamental advancement towards promoting a cost-effective processor-less device-design.

\section{J. IN-NETWORK COMPUTING}

In-Network Computing (INC) is considered as one promising technology to enable high-throughput low-latency computing in industrial IoT systems [32]. With the recent advances in programmable network devices, network devices these days are capable of performing application-specific tasks while still keeping the line-rate forwarding capability. By utilizing this feature, in-network computing paradigm enables the offloading of application-specific tasks from the host to the network devices by utilizing the underlying line-rate processing capacity of the network devices.

As compared to the conventional host-based computing method, in-network computing can provide various advantages. One important advantage of this paradigm is to provide significantly higher throughput processing capacity since it becomes possible to support billions of operations per second (for example, programmable switches have been shown to run at the line-rate of $40 \mathrm{~Gb} / \mathrm{s}$ in [33]) with the help of programmable network devices. Furthermore, another benefit the INC can provide is end-to-end latency minimization in industrial applications as INC can support the processing latency of about sub-microseconds and the transactions can be terminated within the path without the need of rerouting the transmission streams to the edge/cloud servers [32]. Another advantage is in terms of network energy efficiency. In addition to the capability of programmable switches supporting millions of operations per watt [34], network energy consumption caused due to the idle time and the wake-up of devices becomes no longer additional overhead as network devices are already components of the network. Furthermore, higher robustness and lower computational delay are other advantages of distributed processing with the INC as compared to the conventional centralized computing approach [35].

INC can be utilized to enable mobile crowdsensing applications in a context-aware and collaborative manner. Such a collaborative and context-aware INC-enabled crowdsensing can combine the data aggregation at the network-edge, the characteristics of the collected data while extracting the physical context of the crowdsensors across the network, and the context-aware grouping in order to distribute the workload [36]. The performance effectiveness of INC depends on various factors such as the network topology, features of the applications to be supported, load/data distribution across the network, switch architecture and memory capacity. Out of these factors, memory capacity and data distribution are shown to be important parameters which limit the performance of INC in practical networks [37].

\section{CONCLUSIONS}

In this paper, starting with the review of the state-of-the-art of CE, an edge computing-enabled e-URLLC framework for supporting CE devices has been proposed. Various notable enabling techniques/tools for the proposed framework have been thoroughly reviewed. Built on the anticipated evolution of 5G technologies, a notion of "computing as a service" is 
manifested. A low-cost, energy-efficient, and processor-less (minimal processing capability) device-design with hybrid $5 \mathrm{G}$ capabilities is also motivated. Open research topics and future recommendations for the next-generation $\mathrm{CE}$ in the B5G/6G wireless era have been discussed. Various useful recommendations to drive future research work on the nextgeneration CE have been suggested. Furthermore, the potential value creation opportunities for network edge operators through renting of the edge computing facilities with realtime on-demand wireless connectivity on "pay as you go" basis have also been identified.

\section{References}

[1] M. N. Patwary, S. J. Nawaz, M. A. Rahman, S. K. Sharma, M. M. Rashid, and S. J. Barnes, "The potential short- and long-term disruptions and transformative impacts of $5 \mathrm{G}$ and beyond wireless networks: Lessons learnt from the development of a $5 \mathrm{G}$ testbed environment," IEEE Access, vol. 8, pp. 11352-11379, 2020.

[2] GSMA, "The mobile economy," Report, 2019.

[3] P. M. Corcoran, "Cloud computing and consumer electronics: A perfect match or a hidden storm?" IEEE Consumer Electronics Magazine, vol. 1, no. 2, pp. 14-19, 2012.

[4] International Network Generations Roadmap (INGR), “Testbed," IEEE Future Networks: Enabling 5G and Beyond, Report, 2019.

[5] Z. Zhang, Y. Xiao, Z. Ma, M. Xiao, Z. Ding, X. Lei, G. K. Karagiannidis, and P. Fan, "6G wireless networks: Vision, requirements, architecture, and key technologies," IEEE Vehicular Technology Magazine, vol. 14, no. 3, pp. 28-41, 2019.

[6] Technical Specification Group Services and Systems Aspects, "System Architecture for the 5G System; Stage 2, Releases 15," 3GPP, Tech. Rep. document 3GPP TS 23.501, V15.2.0, Jun. 2018.

[7] S. J. Nawaz, S. K. Sharma, S. Wyne, M. N. Patwary, and M. Asaduzzaman, "Quantum machine learning for 6G communication networks: State-ofthe-art and vision for the future,” IEEE Access, vol. 7, pp. 46317-46350, 2019.

[8] J. Aslan, K. Mayers, J. G. Koomey, and C. France, "Electricity intensity of internet data transmission: Untangling the estimates," Journal of Industrial Ecology, vol. 22, no. 4, pp. 785-798, 2018.

[9] I. T. U. (ITU), "IMT traffic estimates for the years 2020 to 2030," Report ITU-R M. 2370-0, ITU-R Radiocommunication Sector of ITU, 2015.

[10] 3GPP, "Study on application architecture for enabling edge applications," TR 23.758,, 2019.

[11] D. Sabella, A. Vaillant, P. Kuure, U. Rauschenbach, and F. Giust, "Mobileedge computing architecture: The role of MEC in the internet of things," IEEE Consumer Electronics Magazine, vol. 5, no. 4, pp. 84-91, 2016.

[12] M. Adil, S. Wyne, and S. J. Nawaz, "On Quantization for Secret Key Generation From Wireless Channel Samples," IEEE Access, vol. 9, pp. $21653-21668,2021$.

[13] S. K. Sharma and X. Wang, "Live data analytics with collaborative edge and cloud processing in wireless IoT networks," IEEE Access, vol. 5, pp. 4621-4635, 2017.

[14] A. Alnoman, S. K. Sharma, W. Ejaz, and A. Anpalagan, "Emerging edge computing technologies for distributed IoT systems," IEEE Network, vol. 33, no. 6, pp. 140-147, 2019.

[15] X. Xu, S. Huang, L. Feagan, Y. Chen, Y. Qiu, and Y. Wang, "EAaaS: Edge analytics as a service," in proc. of IEEE International Conference on Web Services (ICWS), 2017, pp. 349-356.

[16] S. J. Nawaz, S. K. Sharma, B. Mansoor, M. N. Patwary, and N. M. Khan, "Non-coherent and backscatter communications: Enabling ultra-massive connectivity in 6g wireless networks," IEEE Access, vol. 9, pp. 38144 $38186,2021$.

[17] E. Basar, M. Di Renzo, J. De Rosny, M. Debbah, M. Alouini, and R. Zhang, "Wireless communications through reconfigurable intelligent surfaces," IEEE Access, vol. 7, pp. 116 753-116773, 2019.

[18] L. Yang, F. Meng, J. Zhang, M. O. Hasna, and M. D. Renzo, "On the Performance of RIS-Assisted Dual-Hop UAV Communication Systems," IEEE Transactions on Vehicular Technology, vol. 69, no. 9, pp. 10385$10390,2020$.
[19] L. Yang, J. Yang, W. Xie, M. O. Hasna, T. Tsiftsis, and M. D. Renzo, "Secrecy Performance Analysis of RIS-Aided Wireless Communication Systems," IEEE Transactions on Vehicular Technology, vol. 69, no. 10, pp. 12 296-12300, 2020.

[20] M. Adil, S. Wyne, S. J. Nawaz, and B. Muhammad, “Average Contiguous Duration (ACD)-Based Quantization for Secret Key Generation in Generalized Gamma Fading Channels," IEEE Access, vol. 9, pp. 110435 $110450,2021$.

[21] Z. Deng, Q. Li, Q. Zhang, L. Yang, and J. Qin, "Beamforming Design for Physical Layer Security in a Two-Way Cognitive Radio IoT Network With SWIPT," IEEE Internet of Things Journal, vol. 6, no. 6, pp. 10 786-10 798, 2019.

[22] H. Liu, Y. Zhang, and T. Yang, "Blockchain-Enabled Security in Electric Vehicles Cloud and Edge Computing," IEEE Network, vol. 32, no. 3, pp. 78-83, 2018.

[23] I. H. Sarker, M. H. Furhad, and R. Nowrozy, "AI-driven cybersecurity: An overview, security intelligence modeling and research directions," SN Computer Science, vol. 2, no. 3, pp. 1-18, 2021.

[24] S. Li, L. Yang, M. O. Hasna, M.-S. Alouini, and J. Zhang, "Amount of Secrecy Loss: A Novel Metric for Physical Layer Security Analysis,” IEEE Communications Letters, vol. 24, no. 8, pp. 1626-1630, 2020.

[25] S. J. Nawaz, M. Adil, and S. Wyne, "Average Contiguous Duration-A Novel Metric for Characterizing Wireless Fading Channels," IEEE Wireless Communications Letters, vol. 10, no. 8, pp. 1790-1794, 2021.

[26] Q. J. Gu, "THz interconnect: The last centimeter communication," IEEE Communications Magazine, vol. 53, no. 4, pp. 206-215, 2015.

[27] R. Adeogun, G. Berardinelli, P. E. Mogensen, I. Rodriguez, and M. Razzaghpour, "Towards $6 \mathrm{G}$ in-X subnetworks with sub-millisecond communication cycles and extreme reliability," IEEE Access, vol. 8, pp. 110172 $110188,2020$.

[28] B. Varghese, N. Wang, S. Barbhuiya, P. Kilpatrick, and D. S. Nikolopoulos, "Challenges and opportunities in edge computing," in proc. of IEEE International Conference on Smart Cloud (SmartCloud). IEEE, 2016, pp. 20-26.

[29] F. Liu, G. Tang, Y. Li, Z. Cai, X. Zhang, and T. Zhou, "A survey on edge computing systems and tools," Proceedings of the IEEE, vol. 107, no. 8, pp. 1537-1562, 2019.

[30] S. Wang, A. R. Lebeck, and C. Dwyer, "Nanoscale resonance energy transfer-based devices for probabilistic computing," IEEE Micro, vol. 35 no. 5, pp. 72-84, 2015.

[31] K. Camsari and S. Datta, "Dialogue concerning the two chief computing systems: Imagine yourself on a flight talking to an engineer about a scheme that straddles classical and quantum," IEEE Spectrum, vol. 58, no. 4, pp. 30-35, 2021.

[32] T. Mai, H. Yao, S. Guo, and Y. Liu, "In-network computing powered mobile edge: Toward high performance industrial iot,' IEEE Network, vol. 35, no. 1, pp. 289-295, 2021.

[33] T. Jepsen and et al., "Life in the fast lane: A line-rate linear road," in Proc Symposium on SDN Research, 2018, pp. 1-7.

[34] Y. T. et al, "The case for in-network computing on demand," in Proc Fourteenth EuroSys Conf. 2019, 2019, pp. 1-16.

[35] M. Dzaferagic, N. McBride, R. Thomas, I. Macaluso, and N. Marchetti, "Improving in-network computing in iot through degeneracy," IEEE Systems Journal, vol. 15, no. 1, pp. 238-244, 2021.

[36] Y. Du, V. Issarny, and F. Sailhan, "In-network collaborative mobile crowdsensing," in 2020 IEEE International Conference on Pervasive Computing and Communications Workshops (PerCom Workshops), 2020, pp. 1-2.

[37] F. Yang, Z. Wang, X. Ma, G. Yuan, and X. An, "SwitchAgg: A further step towards in-network computing," in proc. of IEEE International Conference on Parallel Distributed Processing with Applications, Big Data \& Cloud Computing, Sustainable Computing \& Communications, Social Computing Networking, 2019, pp. 36-45. 